\title{
Electron-density and energy distributions in a planar inductively coupled discharge
}

\author{
Leonard J. Mahoney, Amy E. Wendt, Ernesto Barrios, Carolyn J. Richards, \\ and J. Leon Shohet \\ Engineering Research Center for Plasma-Aided Manufacturing, University of Wisconsin-Madison, Madison, \\ Wisconsin 53706
}

(Received 24 January 1994; accepted for publication 3 May 1994)

\begin{abstract}
Electron-density and electron energy distribution functions (EEDFs) are measured in a 20-cm-diam by 14-cm-long cylindrical, inductively coupled plasma source driven by fields from a planar, spiral coil at 13.6 MHz. Radio-frequency (rf) -filtered Langmuir probes are used to obtain spatial profilcs of electron population characteristics in argon at powers and pressures of interest for etching and plasma-assisted deposition (1-100 mT). Electron densities range from $10^{10}$ to $10^{12} \mathrm{~cm}^{3}$ with 100 $500 \mathrm{~W}$ of $\mathrm{rf}$ power and peak on axis in the center of the cylindrical volume. The EEDFs show that the observed average electron energy varies by $1-2 \mathrm{eV}$ spatially, with the highest values of average energy occurring at those regions of strongest rf electric field. The EEDF measurements also reveal a significant population of cold electrons trapped in a potential well at the location of peak electron density. From these spatial measurements, spatial estimates of conductivity and ionization rate are deduced.
\end{abstract}

\section{INTRODUCTION}

Several recent reports have demonstrated that planar inductively coupled low-pressure plasma sources are a promising technology for etching and deposition processes where uniform discharges with high plasma density are desired to treat large-area substrates. These sources were first described in patents by Ogle of Lam Research Corporation ${ }^{1}$ and by Coultas and Keller of IBM Corporation ${ }^{2}$ as devices to generate low-pressure, dense discharges for etching and deposition. Design refinements and processing performance for etching have been reported by Keller, Barnes, and Forster ${ }^{3}$ and by Hopwood et al. ${ }^{4-6}$ When optimally designed, these plasma sources are capable of generating uniform plasmas with electron densities on the order of $10^{11}-10^{12} / \mathrm{cm}^{3}$ at 1-10 mTorr in processing gases used for fabricating submicron features. ${ }^{7,8}$

To date, limited empirical characterization of these plasma sources has been made. Hopwood et al. have examined electromagnetic fields, ${ }^{4}$ the uniformity of ion density, ${ }^{5}$ and ion energy distributions (IEDs) ${ }^{9}$ in a planar if induction discharge and Barnes, Forster, and Keller ${ }^{10}$ have examined electron energy distribution functions (EEDFs) of oxygen discharges in a similar system. Much of the aforementioned work has emphasized the uniformity of processing discharges across the work piece, but little work has been performed to determine the spatial discharge characteristics in the interior of these plasma sources. To assist in modeling and designing rf induction plasma sources, we seek to determine the spatial electron population characteristics in the interior of planar inductively coupled argon discharges. This work has been conducted in connection with optical emission studies by Beale, Wendt, and Mahoney ${ }^{11}$ and modeling studies conducted by Ventzek, Hoekstra, and Kushner. ${ }^{12}$ Such information will help to determine discharge heating and diffusion processes in these sources.

\section{EXPERIMENT}

The planar if inductively coupled plasma source used in this work is shown in Fig. 1. The design of this planar coil induction source is similar to inductively coupled plasma sources described by other workers, ${ }^{1-5}$ and has been discussed in recent works regarding pulsed-power operation ${ }^{13}$ and film deposition. ${ }^{14}$ The plasma source and vacuum system is comprised of several components: a vacuum well or recess (1), a four-turn aluminum induction coil (2), a radially spoked Faraday shield (3), a 1.27 -cm-thick by $20.3-\mathrm{cm}$-o.d. quartz vacuum window (4), and a rf impedance matching network (5). The coil current and voltage are monitored by a current transformer (Pearson Model 110) (6) and a calibrated, low-inductance, capacitive voltage divider (7). Gas is fed into the plasma source through an annular gap around the perimeter of the quartz plate (8). The source assembly is mounted on one end of a $61-\mathrm{cm}$-long by $35-\mathrm{cm}$-diam stainless-steel vacuum chamber (9). The coil and quartz vacuum window are recessed into the vacuum chamber for easy access by probes (10) and by optical diagnostics through a $15.2-\mathrm{cm}-i . d$. vacuum view port (11). For the purposes of this work, the main discharge volume is constrained by a $22.8-\mathrm{cm}-\mathrm{i} . \mathrm{d}$. by $13.7-\mathrm{cm}-\mathrm{long}$ grounded, cylindrical, metal liner (12) and an end plate (13). The liner has three $1-\mathrm{cm}$-wide slots cut into its side for access by optical diagnostic systems, and the end plate has a 2.5 -cm-wide slot cut across its diameter for access by the Langmuir probe.

Figure 2 shows the induction coil and Faraday shield as viewed from the vacuum side of the quartz plate. The $1.2 \mu \mathrm{H}$ induction coil has four turns and is $0.17 \mathrm{~cm}$ thick by $1.27 \mathrm{~cm}$ wide with $0.64 \mathrm{~cm}$ spacing between turns. The planar coil is positioned approximately $2 \mathrm{~cm}$ from the discharge/quartz window interface. The grounded, spoked, Faraday shield is used to reduce the degree of capacitive coupling in the source by attenuating divergent if electric fields (capacitive fields) while allowing divergence free electromagnetic fields 


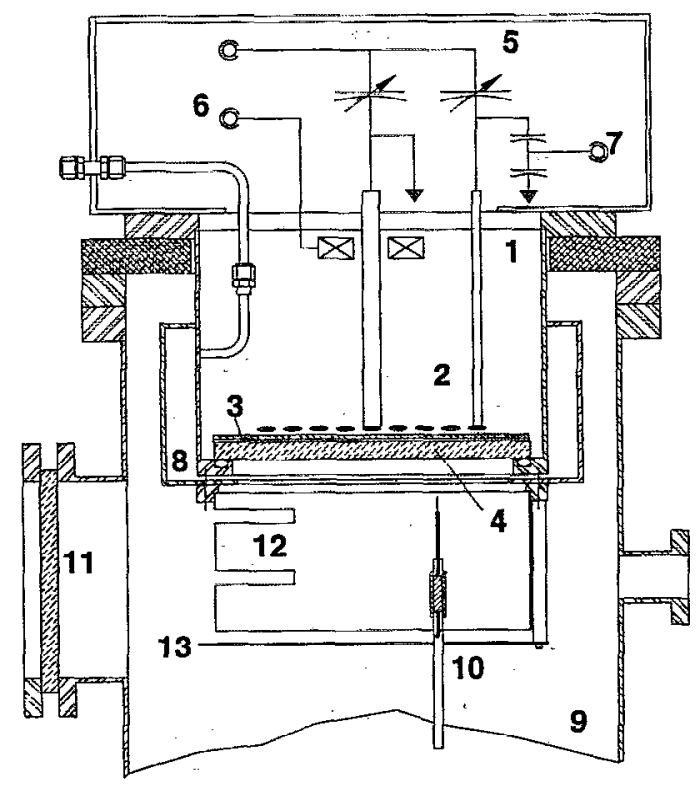

FIG. 1. Planar if induction plasma source: vacuum well (1), induction coil (2), Faraday shield (3), quartz plate (4), impedance matching network (5), current transformer (6), capacitive voltage divider (7), annular gas channel (8), vacuum chamber (9), Langmuir probe (10), optical view port (11), metal liner (12), end plate (13).

(induction ficlds) to pass and heat the discharge. The induction coil and Faraday shield are separated by an insulating glass plate. The spacing and openings in the shield used in this work are designed to allow some penetration of capacitive fields to help initiate the discharge. With the Faraday shield in place, rf potential fluctuations at $13.6 \mathrm{MHz}$ measured with a calibrated capacitive probe placed in the bulk of the plasma are reduced from $10 \mathrm{~V}$ peak to peak to $<2 \mathrm{~V}_{\mathrm{p}-\mathrm{p}}$ and potential fluctuations at the harmonics are reduced to well under $0.05 \mathrm{~V}_{\mathrm{p}-\mathrm{p}}$. Also, when the Faraday shield is in place, both sputtering of the inner ring of the grounded vacuum well near the quartz plate is reduced and localized discharges near open vacuum ports and hollows disappear. These observations indicate that electric fields associated with capacitive coupling are greatly reduced in the vacuum

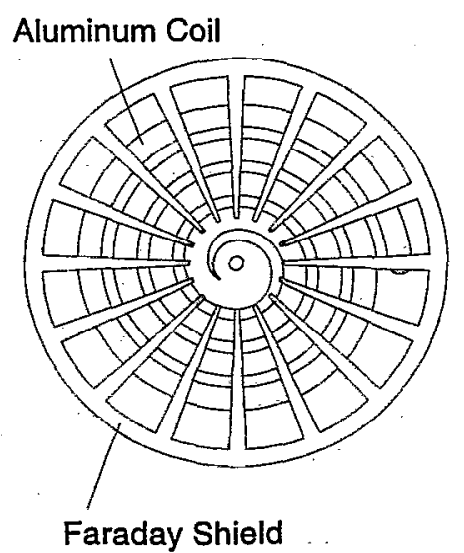

FIG. 2. Bottom view of induction coil and Faraday shield. chamber. Other recent reports and innovations have been made on if inductively coupled plasma sources which feature capacitive shielding components. ${ }^{6,15,16}$

For operation at pressures below $20 \mathrm{mT}$, the vacuum chamber is pumped through a variable conductance throttle valve by an Air Products cryogenic pump. For operation above $20 \mathrm{mT}$, the source is pumped through an automatic throttle valve and cold trap by a 16 cubic feet per minute mechanical pump. Pressure is monitored with a capacitance manometer and/or with an extended range ionization gauge. The vacuum system is also equipped with a UTI $300 \mathrm{AMU}$ Mass Spectrometer which is used to monitor and identify sources of gas impurities during the experiments.

The source is powered at $13.6 \mathrm{MHz}$ by an ENI A-500, $500 \mathrm{~W}$ broadband if power amplifier and a HP model $8116 \mathrm{~A}$ $50 \mathrm{MHz}$ pulse/function generator. The net if power readings are taken as the incident power minus the reflected power as monitored with an in-line watt meter (Bird Electronics model 43), placed between the power supply and impedance matching network. In all of the experimental cases reported here, the impedance matching network is adjusted to minimize reflected power to $<5 \%$ of the forward power. The net if power, coil current, coil voltage, and the variable vacuum capacitor settings are routinely monitored and are used to determine the effective discharge impedance and to help replicate experimental conditions. (It should be noted that coil currents measured at the grounded leg of the coil, as shown in Fig. 1, are typically $17 \%$ greater than those measured at the powered leg for the aluminum coil used in this work.)

The cylindrical Langmuir probe is $7 \mathrm{~mm}$ long and is made from either 10 or 6 mil tungsten wire. To avoid if distortion in the EEDF mcasurements, tuned resonant if filters in combination with a low-pass filter are used to maximize the if impedance of the probe tip to ground. ${ }^{17,18} \mathrm{In}$ addition to the filters, a floating coaxial capacitor is built into the probe tip to reduce the capacitive impedance between the probe tip and the plasma. As a result, the voltage on the probe tip follows rf fluctuations in the plasma potential so that the difference in the potential between the probe and the plasma is constant on the if time scale. The probe body is made of ceramic materials to minimize stray capacitance which often complicates tuning of external if filters. A spectrum analyzer is used to maximize the if impedance of the probe filters at the operating frequency and its first harmonic. Based on the filtering techniques discussed in Ref. 17, the if impedance of the filters is more than adequate to compensate for the rf potential fluctuation levels observed with calibrated capacitive probes.

The EEDF $f_{e}(E)$ is related to the probe $I-V$ characteristics by the Druyvesteyn relation, ${ }^{19}$

$$
\left.f_{e}(E)\right|_{E=e V}=\frac{4}{A_{p} e^{2}}\left(\frac{m_{e} V}{2 e}\right)^{1 / 2} \frac{d^{2} I_{e}}{d V^{2}},
$$

where $E$ is the electron energy, $A_{p}$ is the probe area, $e$ is the electron charge, $m_{e}$ is the electron mass, $V$ is the difference between the plasma potential and probe bias, and $I_{e}$ is the electron current to the probe. Equation (1) is based on several assumptions about the plasma and the probe geometry. The principle assumption is that the local electron velocity 
distribution is isotropic. Another assumption is that the mean free path $\lambda_{\mathrm{mfp}}$, of electrons is large compared to the probe dimensions; otherwise the probe may significantly perturb the discharge. In this work, the probe dimensions are much less than $\lambda_{\mathrm{mfp}}$ up to about $50 \mathrm{~m} \mathrm{~T}$ in argon.

The method used to determine the EEDFs from Langmuir probe measurements is similar to that described by Sudit and Woods ${ }^{20}$ and by Lai et al. ${ }^{21}$ with some minor differences in probe voltage driving circuitry. The probe is swept by a programmable, bipolar power supply (Kepco model $100-2 \mathrm{M})$. The probe current is monitored through a sampling resistor and recorded through a 12 bit digital-to-analog board (Computer Boards CIO-DAS16). To acquire the $I$ - $V$ characteristics, the Langmuir probe is swept from a negative to a positive bias potential over a period of several seconds. To avoid hysteresis effects associated with surface contamination, the probe is held at a negative potential of $-100 \mathrm{~V}$ for cleaning by ion bombardment between sweeps for several seconds. This procedure is adequate to obtain $l-V$ traces which are free of hysteresis effects in this experimental system when using argon.

The EEDFs are obtained by ensemble-averaging over several $I-V$ traces $(10-20)$ and by taking the second derivative of a sliding second-order polynomial fitted to an adjusted window of data points. The plasma potential is identified as the probe voltage at which the second derivative of the $I-V$ trace passes through zero. Using this method of data acquisition and analysis, we obtain $0.3 \mathrm{eV}$ resolution near the plasma potential and a dynamic range of $0-18 \mathrm{eV}$ with two to three decades in the EEDF amplitude. The resolution of the EEDFs at higher energies is limited by the value of the sampling rcsistor and by the input gain and digital resolution of the 12 bit analog-to-digital board. (This limit of the energy resolution is identified by regular positive-to-negative oscillations in the amplitude of the EEDF with increasing energy.) Unless explicitly noted, the electron densities and average electron energies presented in this work have been determined by appropriate numerical integration over the measured EEDFs. Because of the limited range of resolvable electron energy, no attempt is made to account for the effects of sheath expansion in the ion saturation current portion of the $I-V$ trace when evaluating the EEDFs.

\section{RESULTS AND DISCUSSION}

With the metal liner in place, the cylindrical Langmuir probe measurements were taken at several axial and radial positions for several if powers and pressures in argon. Figures 3-5 show spatial contour plots of electron density, plasma potential, and average electron energy, respectively, for a $10 \mathrm{~m} T, 200 \mathrm{~W}$. discharge. Both plasma potential $V_{p}$, and electron density $N_{e}$, are peaked on axis in the system at $z=5 \mathrm{~cm}$. The plasma potential peaks at $18.1 \mathrm{~V}$, and the resulting dc peak ambipolar electric fields are on the order of 1-2 V/cm. The electron density peaks at $19 \times 10^{10} / \mathrm{cm}^{3}$ and drops to about one-tenth of that value near the boundaries of the discharge. The average electron energy $\left\langle E_{e}\right\rangle$ peaks at 5.4 $\mathrm{eV}$ and near $r=6 \mathrm{~cm}$. This location is where the strongest induced of electric fields and currents are believed to occur. ${ }^{4,12} 2-3 \mathrm{~cm}$ away from the quartz plate, the average

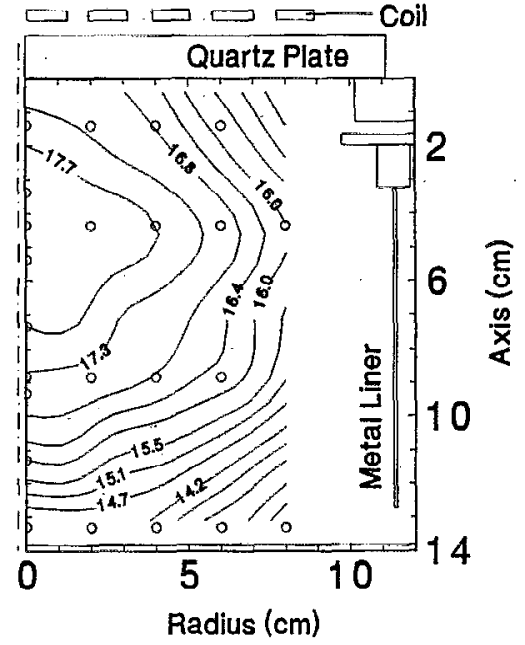

FIG. 3. Spatial contour plot of plasma potential $V_{p}$, for a $10 \mathrm{mT}, 200 \mathrm{~W}$ argon discharge, $V_{p}(\mathrm{peak})=18.1 \mathrm{~V}$. Coil current $=38.6 \mathrm{~A}_{\mathrm{p}-\mathrm{p}}$, coil voltage $=2.85 \mathrm{kV}_{\mathrm{p}-\mathrm{p}}$.

electron energy profile flattens out to a near-constant value of $4.8 \mathrm{eV}$ with only a slight drop off in energy toward the end plate and liner walls.

The spatial contours of electron density, plasma potential, and average electron energy for argon discharges at different powers and pressures are very similar to those depicted in Figs. 3-5. Peak electron densities and plasma potentials occur on axis in the range of $z=4-5 \mathrm{~cm}$, and electron temperatures and average energies peak in the high-rf induction field region of the source between $r=4$ and $6 \mathrm{~cm}$. Table I lists a summary of operating conditions and discharge characteristics for several source conditions. Among these characteristics are bulk $T_{e}$, bulk $\left\langle E_{e}\right\rangle$, and peak $\left\langle E_{e}\right\rangle$. The bulk discharge values are defined as the mean values of $T_{e}$ and $\left\langle E_{e}\right\rangle$ observed at $z>4 \mathrm{~cm}$ within the discharge body, and peak $\left\langle E_{e}\right\rangle$ is the averaged electron energy

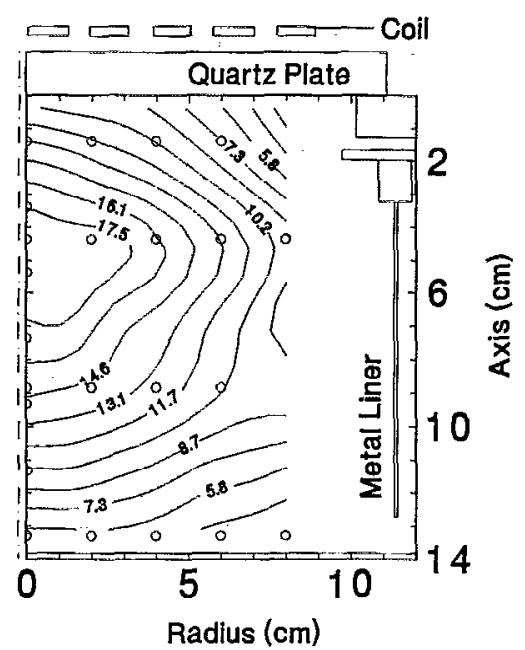

FIG. 4. Spatial profile of electron density $N_{e}$ for a $10 \mathrm{mT}, 200 \mathrm{~W}$ argon discharge, $N_{e}($ peak $)=19 \times 10^{10} / \mathrm{cm}^{3}$. 


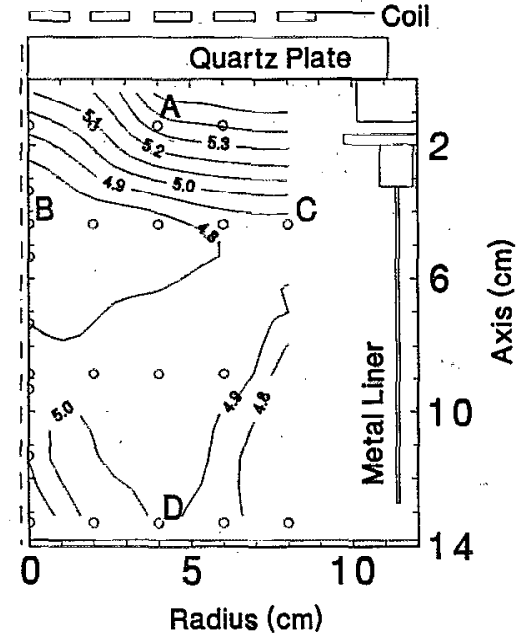

FIG. 5. Spatial profile of average electron energy $\left\langle E_{e}\right\rangle$ for a $10 \mathrm{mT}, 200 \mathrm{~W}$ argon discharge, $T_{e}($ peak $)=5.4 \mathrm{eV}$.

measured at $(r, z)=(4,1.4) \mathrm{cm}$. Coil current and voltage readings are also reported for these cases.

Figure 6. shows several normalized EEDFs for points indicated in the $\left\langle E_{e}\right\rangle$ contour plot of Fig. 5. The EEDFs appear to be Maxwellian, but clearly there is significant spatial variation in the form of the EEDFs. At $10 \mathrm{mT}$, we see that the EEDFs measured in the interior of the discharge exhibit a cold distribution of electrons with $T_{e}$ (cold) $=0.7 \mathrm{eV}$ imposed on a bulk electron distribution with $T_{e}$ (bulk) $=3.4$ $\mathrm{eV}$. These cold electrons are concentrated about the peak of the plasma potential and are trapped by the ambipolar potential well of the discharge. We also observe that the fraction of cold electrons increases with power and pressure and cleanliness of the system. Figure 7 shows several normalized EEDFs taken in a $50 \mathrm{mT}$ discharge at various radial positions and $z=4.4 \mathrm{~cm}$. Once again, a population of cold electrons, here $T_{e}$ (cold) $=0.3 \mathrm{eV}$, is observed at the center of the discharge body.

The same EEDF data used to determine the plasma characteristics may be used to calculate discharge transport coefficients and electron impact collision rates. For example, the definition of electrical conductivity $\sigma$, assuming a uniform neutral gas pressure and locally uniform, isotropic electron velocity distribution $f_{0}$, is 22

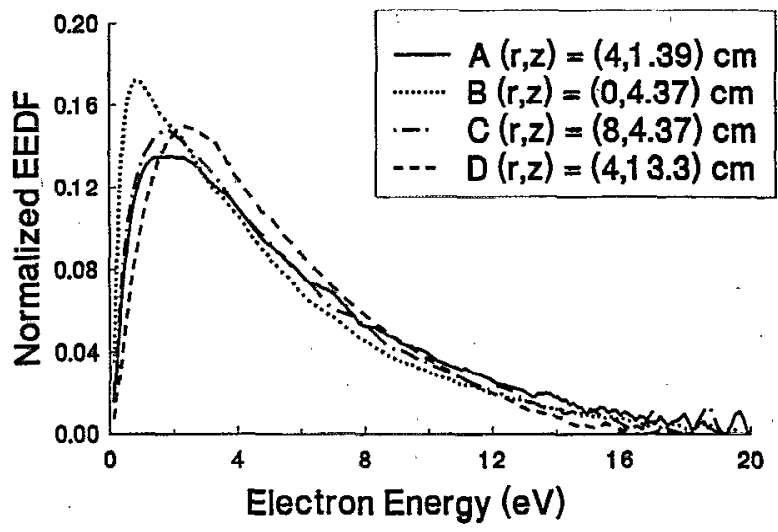

FIG. 6. Normalized EEDFs for several spatial points in a $10 \mathrm{mT}, 200 \mathrm{~W}$ argon discharge, as indicated in Fig. 5.

$$
\sigma=-\frac{4 \pi e^{2}}{3 m_{e}} \int \frac{v^{3}\left(\partial f_{0} / \partial v\right)}{\nu_{m}(v)+j \omega} d v
$$

where $m_{e}$ is the electron mass, $v$ is the electron velocity, $\nu_{m}$ is the collision frequency for momentum transfer, and $\omega$ is the frequency of the time-harmonic electric field. If it is assumed that $\nu_{m}$ is independent of velocity, Eq. (1) reduces to

$$
\sigma=\frac{N_{e} e^{2}}{m_{e}}\left(\frac{1}{\nu_{m}+j \omega}\right)
$$

which is the expression of ac electrical conductivity widely used in the analysis of ohmically heated discharges. Using momentum-transfer-collision cross-section data from Frost and Phelps ${ }^{23}$ and measurements of EEDFs for the $10 \mathrm{mT}$, $200 \mathrm{~W}$ case, the real and imaginary part of the conductivity are calculated for Eq. (2) and are shown in Figs. 8(a) and 8(b). The electrical conductivity clearly follows trends in the electron density. This result is expected as the values for $\nu_{m}$ (around $3.6 \times 10^{7} \mathrm{~s}^{-1}$ ), are less than half that of $\omega$ and only vary $10 \%$ within the discharge volume. Conductivity values calculated using Eq. (3) are $10 \%$ lower than those calculated with Eq. (2) and exhibit nearly the same spatial trends. Ionelectron collisions were also considered in the calculation of the conductivity, but were not found be significant at the $10^{10}-10^{11} / \mathrm{cm}^{3}$ density levels observed at these conditions.

TABLE I. Characteristic values of measured discharge parameters for various power and pressure settings.

\begin{tabular}{ccccccccc}
\hline \hline $\begin{array}{c}\text { Pressure } \\
(\mathrm{mT})\end{array}$ & $\begin{array}{c}\text { Net if } \\
\text { power }(\mathrm{W})\end{array}$ & $\begin{array}{c}\text { Coil current } \\
\left(\mathrm{A}_{\mathrm{p}-\mathrm{p}}\right)\end{array}$ & $\begin{array}{c}\text { Coil voltage } \\
\left(\mathrm{kV}_{\mathrm{p}-\mathrm{p}}\right)\end{array}$ & $\begin{array}{c}\text { Peak } N_{e} \\
\left(10^{10} / \mathrm{cm}^{3}\right)\end{array}$ & $\begin{array}{c}\text { Peak } V_{p} \\
(\mathrm{~V})\end{array}$ & $\begin{array}{c}\text { Peak }\left\langle E_{e}\right\rangle \\
(\mathrm{eV})\end{array}$ & $\begin{array}{c}\text { Bulk } T_{e} \\
(\mathrm{eV})\end{array}$ & $\begin{array}{c}\text { Bulk }\left\langle E_{e}\right\rangle \\
(\mathrm{eV})\end{array}$ \\
\hline $10^{\mathrm{a}}$ & 100 & 30.8 & 2.27 & 9.9 & 17.9 & 5.3 & 3.5 & 4.7 \\
$10^{\mathrm{a}}$ & 200 & 38.6 & 2.85 & 19.0 & 18.1 & 5.4 & 3.5 & 4.8 \\
$20^{\mathrm{a}}$ & 100 & 28.8 & 2.20 & 19.8 & 16.7 & $\cdots$ & 2.8 & 3.9 \\
$20^{\mathrm{a}}$ & 200 & 37.4 & 2.78 & 38.3 & 16.0 & $\ldots$ & 2.6 & 3.7 \\
$20^{\mathrm{b}}$ & 100 & 29.0 & 2.12 & 18.2 & 17.3 & $\ldots$ & 3.2 & 4.2 \\
$20^{\mathrm{b}}$ & 200 & 38.2 & 2.71 & 39.4 & 17.1 & 4.7 & 2.9 & 4.1 \\
$50^{\mathrm{b}}$ & 100 & 26.6 & 2.00 & 50.0 & 16.0 & 3.9 & 2.3 & 3.5 \\
$100^{\mathrm{b}}$ & 100 & 25.6 & 1.86 & 55.3 & 14.4 & 3.5 & 1.9 & 3.1 \\
\hline \hline
\end{tabular}

The vacuum system was operated with a throttled cryogenic pump.

${ }^{b}$ Ihe vacuum system was operated with a throttled mechanical pump. 


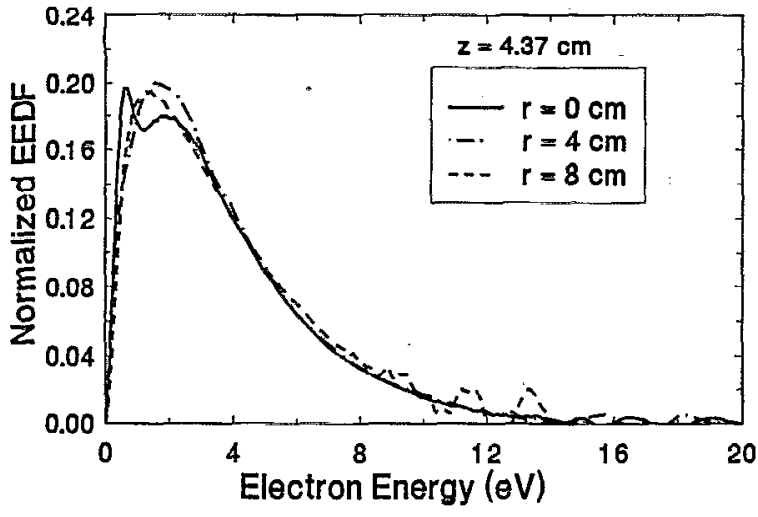

FIG. 7. Normalized EEDFs observed along the radius of a $50 \mathrm{mT}, 100 \mathrm{~W}$, argon discharge at $4.4 \mathrm{~cm}$ from the quartz plate.

These values of complex conductivity may be used to approximate the depth of penetration of the rf electric fields. The skin depth of the fields $\delta$ may be approximated by

$$
\delta \approx \frac{1}{\operatorname{Re}\left(\sqrt{\omega \mu_{0} j\langle\sigma\rangle}\right)},
$$

where $\mu_{0}$ is the permeability of free space and $\langle\sigma\rangle$ is the average value of conductivity along the discharge length at a fixcd radius. Equation (4) reduces to the standard expression for the collisional skin depth when $\nu_{m} \gg \omega$ and to the collisionless skin depth when $v_{m} \ll(\omega)$. For the conductivity values shown Figs. 8(a) and 8(b), the skin depth at $R=5 \mathrm{~cm}$ is on the order of $4.0 \mathrm{~cm}$. This depth of penetration of the rf fields is reflected in the gradients of electron temperature observed in Fig. 5 and is consistent with the work of Ref. 4 where direct measurements of the attenuation of if magnetic fields in low-pressure inductively coupled argon discharges were found to range from 1.6 to $3.6 \mathrm{~cm}$. The estimate of $\delta$ is also consistent with two-dimensional hybrid modeling work discussed in Ref. 12. The above analysis is only approximate as the actual falloff of the rf field is not purely exponential because of the inhomogeneous conductivity of the discharge.

Considering the spatial variation in the EEDFs, rates of electron collision processes which are sensitive to highenergy electrons in the tail of the EEDF may exhibit spatial variation in the discharge body that is significantly different from that of the electron-density profile. To facilitate the calculation of inelastic collision rates, statistical fits of analytical EEDFs are made to measured EEDFs. Using the nonlinear regression procedures (the Bates and Watts NLS functions), ${ }^{24}$ in the S-PLUS program, ${ }^{25}$ we fit the following function to measured EEDFs:

$$
f_{e}(E)=a \sqrt{E} \exp \left(-b E^{x}\right),
$$

where $x=1$ for a Maxwellian electron energy distribution. An example of a fit is shown in the semilog plot in Fig. 9. Such fits are used to estimate the EEDFs at electron energy levels greater than can be readily resolved by the Langmuir probe diagnostic system, which is around $16-18 \mathrm{eV}$.

Figure 10 shows the single-step ionization rate for argon as calculated from ionization collision cross-section data
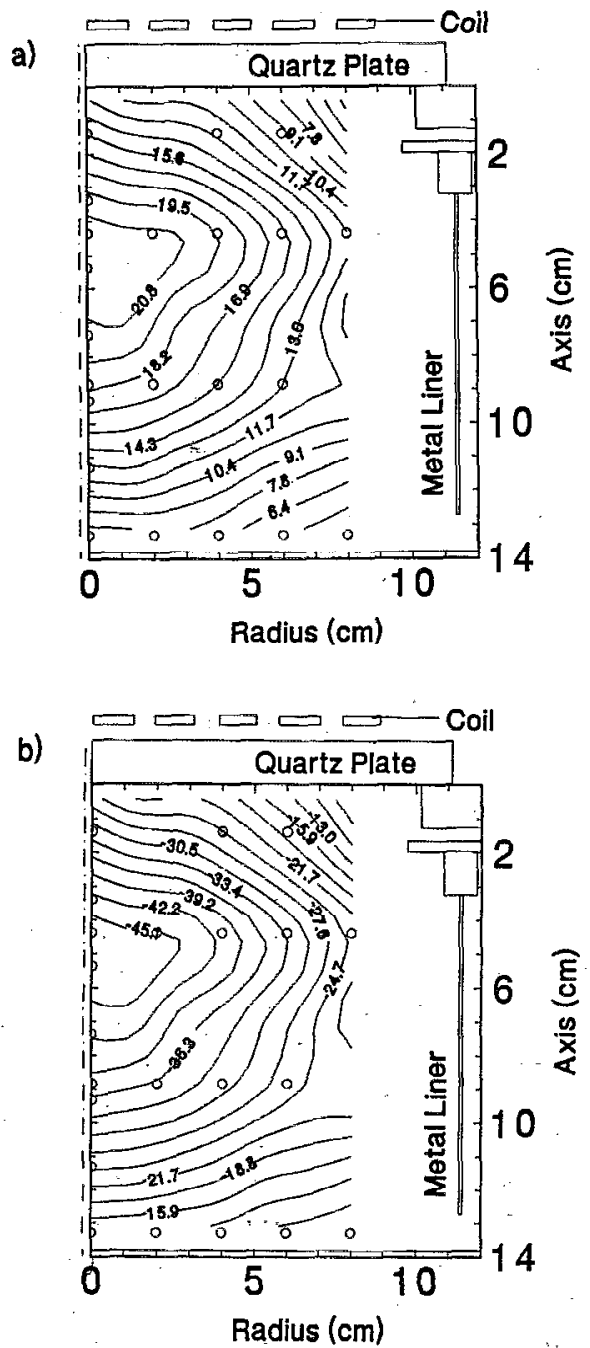

FIG. 8. Electrical conductivity for a $10 \mathrm{mT}$ argon discharge at $200 \mathrm{~W}$; (a) real part of conductivity, $\sigma$ (peak) $=21 \mathrm{~S} / \mathrm{m}$; (b) imaginary part of conductivity, $\sigma($ peak $)=-j 46 \mathrm{~S} / \mathrm{m}$.

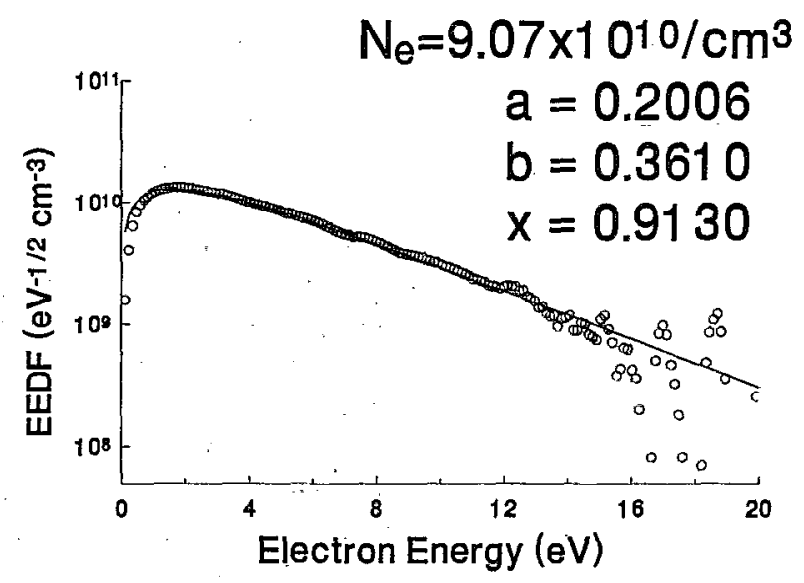

FIG. 9. Example of nonlinear regression analysis of EEDFs used to extrapolate the EEDFs to higher-energy values. 


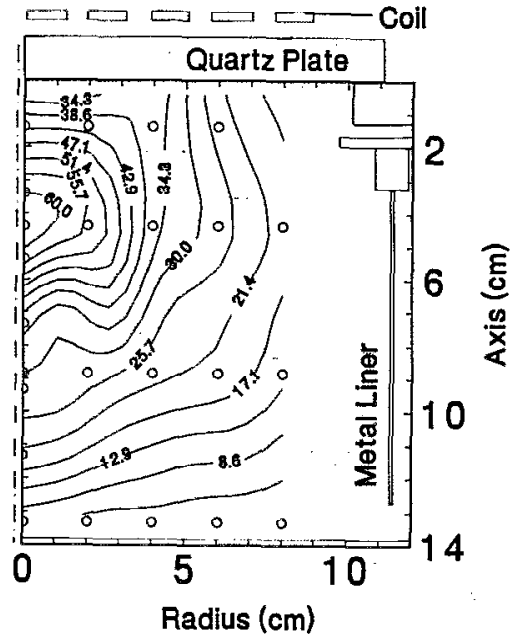

FIG. 10. Spatial profile of single-step ionization rate for the $10 \mathrm{mT}, 200 \mathrm{~W}$ argon discharge, peak ionization rate $=61 \times 10^{15} /\left(\mathrm{cm}^{3} \mathrm{~s}\right)$.

from Rapp and Englander-Golden. ${ }^{26}$ These calculations suggest that the single-step ionization rate is three times greater at the quartz boundary than at the end plate and is peaked where the electron density peaks, near the center of the discharge. Similar trends are to be expected with other collision rates which are sensitive to the tail of the EEDF, such as excitation of argon neutrals from their ground state to emitting and metastable states. However, the spatial profile of single-step ionization in Fig. 10 appears to conflict with spatially resolved optical emission measurements of the $1 s 5$ $2 p 10$ and $1 s 3-2 p 7$ (Paschen notation) neutral argon transitions performed in a related investigation. ${ }^{11}$ These measurements show a ring-shaped structure, suggesting that the electron impact excitation rate (and therefore the singlestep ionization rate) has an off-axis maximum in the region of peak electric field. The apparent discrepancy between spatial profiles of single-step ionization rate calculations and neutral excitation rates inferred from the optical emission measurements may be attributed to assumptions made in performing the EEDF measurements.

An implicit assumption made in the measurements of the EEDFs, and in the calculations of conductivity and ionization rate, is that the electron velocity distribution in nearly isotropic; however, this may not be the case. Due to the relatively large of drift currents in the high-induction-field region close to the quartz plate, the electrons in this region experience a strong if azimuthal drift. Should the drift velocity amplitude be significant in comparison to the thermal velocity, then the assumption of an isotropic velocity distribution used in evaluating the $I-V$ probe traces may be invalid and the reported values of the discharge characteristics, conductivity, and ionization rate may be inaccurate. Another possible contributor to this discrepancy is the use of statistical fits of Eq. (5) to estimate the level of high-energy electrons in the tail of the EEDFs, which cannot be resolved by the Langmuir probe diagnostic system. There could be spatial variation in the tail of the distributions that is not captured by the fit.

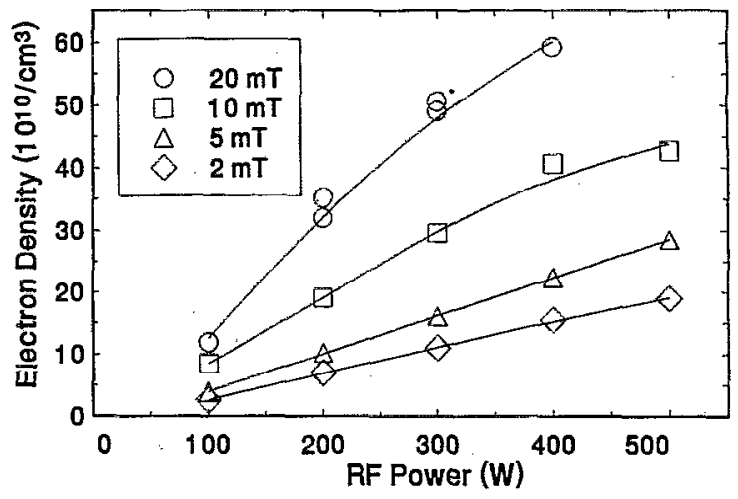

FIG. 11. Electron density vs net if power at various pressure levels measured at $r=0 \mathrm{~cm}$ and $z=5.0 \mathrm{~cm}$.

Figure 11 shows electron-density measurements taken at various low pressures and net if powers in an argon discharge with the metal liner in place and the probe located at $z=5.0 \mathrm{~cm}$, which is near the peak density location in the discharge. (Note: The electron densities shown in this figure are calculated by conventional probe analysis through the determination of the electron saturation current and estimation of an electron temperature. ${ }^{27}$ ) Electron densities range from $10^{10}$ to $10^{12}$ electrons $/ \mathrm{cm}^{3}$. These electron density values are two to five times lower than ion densities reported by workers characterizing similar planar inductively coupled plasma source systems; $;, 3,7$ however, this is to be expected because electron-density measurements based on electron saturation currents or integration of the EEDF are normally two to five times lower than ion densities determined from ion saturation current readings even for electropositive discharges. 28

In Fig. 11, $N_{e}^{-}$is seen to increase in direct proportion to the discharge pressure and power, although it appears to eventually saturate as seen in the $20 \mathrm{mT}$ case. At this pressure, the saturation in density may be explained by the decrease in the skin depth with the increase in electron density. As the level of if power and electron density is increased, the skin depth decreases, and as a result the effective plasma resistance and power coupling efficiency also decrease. After reaching saturation in the electron density, further increases in rf power may be dissipated in the induction coil and impedance matching network. This scenario has been discussed by Piejak, Godyak, and Alexandrovich. ${ }^{29}$ Also, Hopwood et al. ${ }^{5}$ observed a similar saturation in ion densities in very low-pressure $(\$ 1 \mathrm{mT})$, highly dense krypton discharges, and suggested that the "nonlinear" behavior at low pressure may be attributed to neutral depletion of the highly ionized gas.

\section{SUMMARY}

Spatial measurements of argon discharge characteristics have been performed in an experimental planar if inductively coupled plasma source. Over the power and pressure range examined, electron density $\left(10^{10}-10^{12} / \mathrm{cm}^{3}\right)$ and plasma potentials are peaked on axis, suggesting that spatial profiles of the argon discharge characteristics in the given source geom- 
etry are diffusion dominated. The EEDFs reveal a significant population of cold electrons trapped in the ambipolar potential field about the center of the discharge. Inhomogeneities in the spatial profiles of averaged electron energy are observed with peak energies and energy gradients located within $2-3 \mathrm{~cm}$ from the quartz plate. These spatial features in the electron energy appear to be coincident with the radial profile and skin depth of the rf heating fields.

Based on the measured EEDFs, single-step ionization rates for argon are calculated and show single-step ionization to be peaked on-axis. This result is in apparent conflict with spatially resolved optical emission measurements, reported elsewhere, ${ }^{11}$ in which the emission intensity of excited of neutral argon atoms exhibits an off-axis peak in the source. The discrepancy may be due to an incorrect assumption of an isotropic electron velocity distribution made in the measure of EEDFs. Strong azimuthal currents in the rf power coupling regions of the plasma source may result in anisotropic electron velocities and give rise to collision rates that are not accurately determined from the Langmuir probe measurements made in this work.

\section{ACKNOWLEDGMENTS}

We thank Dr. Isacc Sudit (Department of Electrical Engineering at UCLA) for consultation on EEDF measurement techniques. We also thank Dr. Jeffrey Hopwood (Department of Electrical and Computer Engineering at Northeastern University) and Dr. Peter Ventzek and Dr. Mark Kushner (Department of Electrical and Computer Engineering at the University of Illinois, Urbana) for their many thoughtful discussions on planar rf inductively coupled plasma sources. This work is supported by the National Science Foundation under Grant No. ECD-8721545.

\footnotetext{
${ }^{1}$ J. S. Ogle, U.S. Patent No. 4,948,458 (14 August 1990).

${ }^{2}$ D. K. Coultas, and J. H. Keller, European Patent, Publication No. 0379 828 A2 (8 January 1990).
}

${ }^{3}$ J. H. Keller, S. M. Barnes, and J. C. Forster, 43rd Gaseous Electronics Conference, Champaign-Urbana, IL, 1990.

${ }^{4}$ J. Hopwood, C. R. Guarnieri, S. J. Whitehair, and J. J. Cuomo, J. Vac. Sci. Technol. A 11, 147 (1993).

${ }^{5}$ J. Hopwood, C. R. Guarnieri, S. J. Whitehair, and J. J. Cuomo, J. Vac. Sci. Technol. A 11, 152 (1993).

'J. Hopwood, Plasma Sources Sci. Technol. (to be published).

${ }^{7}$ R. Patrick, P. Schoenborn, H. Toda, and F. Bose, J. Vac. Sci. Technol. A 11, 1296 (1993).

${ }^{8}$ J. B. Carter, J. P. Holland, E. Peltzer, B. Richardson, E. Bogel, H. T. Nguyen, Y. Melaku, D. Gates, and M. Ben-Dor, J. Vac. Sci. Technol. A 11, 1301 (1993).

${ }^{9}$ J. Hopwood, Appl. Phys. Lett. 62, 940 (1993).

${ }^{10}$ M. S. Barnes, J. C. Forster, and J. H. Keller, Appl. Phys. Lett. 62, 2622 (1993).

${ }^{11}$ D. F. Beale, A. E. Wendt, and L. J. Mahoney, J. Vac. Sci. Technol. A (to be published).

${ }^{12}$ P. L. G. Ventzek, R. J. Hoekstra, and M. J. Kushner, J. Vac. Sci. Technol. A 12, 461 (1994).

${ }^{13}$ A. E. Wendt, L. J. Mahoney, and J. L. Shohet (unpublished).

${ }^{14}$ J. A. Tobin, G. Li, L. J. Mahoney, D. D. Denton, and J. L. Shohet, Plasma Chem. Plasma Proc. (to be published).

${ }^{15}$ J. W. Denneman, J. Phys. D 23, 293 (1990).

${ }^{16}$ W. L. Johnson, U.S. Patent No. 5,234,529 (10 August, 1993).

${ }^{17}$ R. R. J. Gagné and A. Cantin, J. Appl. Phys. 43, 2639 (1972).

${ }^{18} \mathrm{~V}$. A. Godyak, in Plasma Surface Interactions and Processing of Materials, ediled by $\mathrm{O}$. Auciello, A. Gras-Marti, J. A. Valles-Abarca, and D. L. Flamm (Kluwer, Boston, 1990), pp. 95-134.

${ }^{19}$ M. J. Druyvesteyn, Z. Phys. 64, 781 (1930).

${ }^{20}$ I. D. Sudit and R. C. Woods, Rev. Sci. Instrum. 64, 2440 (1993).

${ }^{21}$ C. Lai, R. A. Breun, P. W. Sandstrom, A. E. Wendt, N. Hershkowitz, and R. C. Woods, J. Vac. Sci. Technol. A 11, 1199 (1993).

${ }^{22}$ B. E. Cherrington, Gaseous Electronics and Gas Lasers (Perganom, New York, 1979), pp. 95-97.

${ }^{23}$ L. S. Frost and A. V. Phelps, Phys. Rev. A 136, 1538 (1964).

${ }^{24}$ D. Bates and D. Watts, Nonlinear Regression Analysis and Its Applications (Wiley, New York, 1988), p. 108.

${ }^{25}$ s-PLus, Version 3.1, Statistical Sciences Inc., Seattle, WA, 1993.

${ }^{20}$ D. Rapp and P. Englander-Golden, J. Chem. Phys. 43, 1464 (1965).

${ }^{27}$ J. D. Swift and M. J. R. Schwar, Flectric Probes for Plasma Diagnostics (American Elsevier, New York, 1969), pp. 1-15.

${ }^{28}$ I. D. Sudit, Ph.D. thesis, University of Wisconsin-Madison, 1992.

${ }^{29}$ R. B. Piejak, V. A. Godyak, and B. M. Alexandrovich, Plasma Sources Sci. Technol. 1, 36 (1992). 